\title{
Effects of physical activity on quality of life of coronary artery bypass grafted patients: Re-examining the evidence from randomized clinical trials through systemic review
}

\author{
ying mao $^{1}$, Ling $\operatorname{sun}^{1}$, and Jiequn Zhang $^{1}$ \\ ${ }^{1}$ 1. Heart Center, Wuxi Mingci Cardiovascular Hospital, Wuxi, PR China
}

May 18, 2020

\begin{abstract}
Objective: Coronary artery disease (CAD) is associated with remodeling and narrowing of the coronary arteries and is the primary cause of mortality and handicap around the globe. Coronary artery bypass grafting (CABG) is an established method for the treatment and symptomatic relief of severe form of CAD. Post-GABG patients experience poorer quality of life and worse long-term psychological outcomes. This study systemically evaluates the effects of physical activity intervention on the quality of life of post GABG patients. Methods: Two randomized controlled trials recruiting 120 post-CABG patients met the inclusion criteria and were systemically analyzed. Endpoints measured in this study included physical and mental components related to quality of life. Data was analyzed through selected statistical tool while using mean difference and $95 \%$ confidence interval for analysis. Results: Mild to moderate physical activity in the form of exercise or physiotherapy intervention for 4 to 24 weeks improved the scores of QoL in post-CABG patients in intervention group (36.41 \pm 5.12$)$ as compared to control group $(25.39 \pm 6.22)$ at the end of follow up period. In comparison to control group, scores of both physical and mental components including psychological role, physical function, general health, bodily pain, social function, vitality, and mental health were improved for post-CABG patients in intervention group. Conclusion: Results reveal that mild to moderate physical activity can improve quality of in post-GABG patients moderate manner, however, further investigation in large population size in needed prior to recommending physical activity in rehabilitation programs of the target patients.
\end{abstract}

\section{Hosted file}

manuscript.docx available at https://authorea.com/users/322628/articles/452167-effectsof-physical-activity-on-quality-of-life-of-coronary-artery-bypass-grafted-patients-reexamining-the-evidence-from-randomized-clinical-trials-through-systemic-review 